\title{
Determination of Optimal Non-Denaturing Elution Conditions from Affinity Columns by a Solid-Phase Screen
}

BioTechniques 31:584-596 (September 2001)

\author{
Hendrik Fuchs, Rudolf \\ Tauber, and Reinhard \\ Geßner ${ }^{1}$ \\ Freie Universität Berlin, and \\ ${ }^{1}$ Humboldt-Universität zu \\ Berlin, Berlin, Germany
}

\section{INTRODUCTION}

Preparative purification of proteins relies predominantly on column chromatography. For the development of a particular protein purification procedure, five or more different columns are frequently used. Once the protein has been purified, the method is commonly simplified by introducing a single highaffinity column specific for the target protein. This affinity column can be prepared by immobilizing specific antibodies, ligands, or, in the case of enzymes, often inhibitors to a solid-phase matrix. A high affinity of the protein for the matrix reduces the number of purification steps but increases the risk of destroying the protein's function because strong denaturing conditions, such as highly chaotropic salts, detergents, or a low $\mathrm{pH}$, are usually required to dissociate a tightly bound protein from the column.

A well-known example for highaffinity chromatography is the purification of the transferrin receptor (TfR) using a transferrin-sepharose column (11). However, elution with $500 \mathrm{mM}$ $\mathrm{NaSCN}$ may produce a fraction that aggregates during gel filtration (11), and elution with $500 \mathrm{mM} \mathrm{KSCN}$ leads to a considerable fraction of receptor molecules that are unable to rebind to transferrin even after dialysis against a 10$\mathrm{mM}$ phosphate buffer containing 150 $\mathrm{mM} \mathrm{NaCl}$ (data not shown).

Previously, determining the best elution conditions required sequential testing of various buffers at a number of different concentrations on a repeatedly loaded affinity column. In addition to the large amount of crude extract required, this procedure risks irreversibly inactivating the affinity column by using elution conditions that are too harsh. In the present work, we describe a new solid-phase assay for the fast determination of optimal conditions for ligand elution (DOCILE). The assay also allows one to analyze whether the chosen conditions will permit retention of the binding activity of the affinity column.

The TfR mediates the cellular uptake of iron by binding the serum iron transport protein transferrin with high affinity $\left[\left(K_{\mathrm{D}}=0.12-0.16 \mathrm{nM}\right)(2)\right]$. The human TfR is composed of two identical disulfide-linked subunits that bind one transferrin molecule each (8). After internalization of the complex and intracellular iron release, the complex cycles back to the cell surface where apo-transferrin becomes replaced by ferri-transferrin $(3,7)$. Therefore, the transferrin receptor can be purified on a ferri-transferrin affinity column, which can serve as a model system for the verification of this new assay.

\section{MATERIALS AND METHODS}

\section{Materials}

Desferrioxamine mesylate and human apo-transferrin were purchased from Sigma (St. Louis, MO, USA), monoclonal rabbit anti-mouse $\kappa$-chain antibodies (clone H139.52.1) were 
from Dianova GmbH (Hamburg, Germany), and monoclonal mouse antitransferrin antibodies MAB033-19/1 were from Chemicon International (Temecula, CA, USA). Monoclonal mouse anti-TfR antibodies OKT9 were prepared from a hybridoma cell line as previously described (4). Fetal calf serum (FCS) was obtained from Invitrogen (Carlsbad, CA, USA), CNBr-activated Sepharose ${ }^{\circledR}$ 4B was from Amersham Pharmacia Biotech (Uppsala, Sweden), 3,3',5,5'-tetramethylbenzidine was from Merck (Darmstadt, Germany), and octylpolyoxyethylene (8POE) was from Bachem Biochemica GmbH (Heidelberg, Germany).

\section{Preparation of a Ferri-Transferrin Affinity Column}

Human apo-transferrin was saturated with $\mathrm{Fe}^{3+}$ according to Karin et al. (9). However, the PBS (150 mM NaCl and $10 \mathrm{mM}$ phosphate, $\mathrm{pH} 7.5$ ) was supplemented with $1 \mathrm{mM}$ sodium bicarbonate because we found that, in this case, saturation occurred more than 10 times faster ( $15 \mathrm{~min}$ instead of $3 \mathrm{~h}$ ), and the equilibrium was shifted toward the iron-saturated form (data not shown). Coupling of ferri-transferrin to CNBr-activated sepharose was performed according to the manufacturer's instructions.

\section{Purification of the TfR}

Human TfR was solubilized and separated from bound transferrin as previously described (11). The solubilized receptor was loaded onto a ferritransferrin sepharose affinity column. The column was pre-eluted with PBS $(\mathrm{pH} 7.5)+1 \%$ 8-POE $+2 \mathrm{M} \mathrm{KCl}$ to remove TfR bound with only one binding site to immobilized ferri-transferrin and saturated on the other with soluble transferrin originating from the preparation. After pre-elution, the column was washed with $0.2 \mathrm{mM}$ desferrioxamine mesylate in $10 \mathrm{mM}$ citrate, $\mathrm{pH}$ 5.0, and $150 \mathrm{mM} \mathrm{NaCl}$ to remove iron from transferrin and therefore reduce the affinity between the transferrin and its receptor. After re-equilibration with PBS ( $\mathrm{pH} 7.5)+1 \%$ 8-POE, highly purified, transferrin-free TfR was eluted with PBS $(\mathrm{pH} 7.5)+1 \%$ 8-POE + $2 \mathrm{M}$
$\mathrm{KCl}, \mathrm{pH}$ 7.5. The samples were desalted on PD-10 columns (Amersham Pharmacia Biotech) equilibrated with PBS and $1 \%$ 8-POE and stored at $4{ }^{\circ} \mathrm{C}$.

\section{DOCILE}

We designed this assay to be performed in modular 96-well microplates at the desired temperatures $\left(4^{\circ} \mathrm{C}-\right.$ $\left.25^{\circ} \mathrm{C}\right)$. All of the experiments presented here were done at $21^{\circ} \mathrm{C}$. Sample volumes were $100 \mu \mathrm{L} /$ well unless otherwise noted. The wash procedures between any two successive incubation steps included three wash steps with $250 \mu \mathrm{L}$ PBST $\left(\mathrm{PBS}+0.05 \%\right.$ Tween ${ }^{\circledR}$ 20 ), followed by a 5-min incubation with $150 \mu \mathrm{L}$ PBST, and then another three $250-\mu \mathrm{L}$ PBST wash steps. Upon incubation, the microplates were constantly agitated in a Varishaker Incubator at medium speed (Thermo Labsystems, Helsinki, Finland).

DOCILE was performed with either immobilized ligand protein (ferri-transferrin) or immobilized target protein (TfR). TfR (100 ng/well) or ferri-transferrin (500 ng/well) was incubated in Nunc-immuno ${ }^{\mathrm{TM}}$ modules MaxiSorp ${ }^{\mathrm{TM}}$ U16 (Nalge Nunc International, Roskilde, Denmark) in PBS for $1.5 \mathrm{~h}$. Excess binding sites were blocked for 30 min with $200 \mu \mathrm{L}$ PBS containing $3 \%$ BSA and $10 \%$ FCS. Subsequently, 50 ng ferri-transferrin (in case of TfR coating) or $25 \mathrm{ng}$ TfR (in case of transferrin coating) were incubated in PBST for 2.5 $h$. Thereafter, the samples were incubated for $30 \mathrm{~min}$ with $16 \mathrm{ng} /$ well monoclonal antibodies MAB033 against human transferrin (in case of TfR coating) or with anti-TfR antibody OKT9 (in case of transferrin coating) in PBST. Both primary antibodies were then incubated for $30 \mathrm{~min}$ with $5 \mathrm{ng} /$ well peroxidase-conjugated monoclonal rabbit antimouse $\kappa$-chain antibodies in PBST. For the following enzyme-catalyzed reaction, a $0.02 \%$ solution of $3,3^{\prime}, 5,5^{\prime}$ tetramethylbenzidine in $40 \mathrm{mM}$ potassium citrate buffer, $\mathrm{pH} 3.95$, containing $0.01 \% \mathrm{H}_{2} \mathrm{O}_{2}$ was used (6). The reaction was stopped after $10 \mathrm{~min}$ by the addition of $50 \mu \mathrm{L} 2 \mathrm{M} \mathrm{H}_{2} \mathrm{~S} \mathrm{O}_{4}$. Absorbance was measured at $450 \mathrm{~nm}$ in an MR 7000 multichannel photometer (Thermo Labsystems) and was corrected for light- scattering effects by subtracting the absorbance at $490 \mathrm{~nm}$.

Two common elution agents, KSCN and urea, were tested for DOCILE. Both the dissociation caused by the suppression of the binding forces (elution experiment) and the dissociation that results from irreversible denaturation of the binding partners (stability experiment) were measured. Experimentally, these cases were distinguished by $(i)$ incubating the solubilized TfR ( $25 \mathrm{ng} /$ well) in the presence of various concentrations (indicated in Figures 1-4) of KSCN or urea (transferrin was coated) and by (ii) preincubating the immobilized TfR before the transferrin binding step (and before blocking) for $90 \mathrm{~min}$ with identical concentrations of the respective salt in PBST. In the latter case, the high salt was removed before the binding assay as previously described.

To check the effect of the eluent on the binding activity of the affinity column, immobilized transferrin was preincubated before TfR binding (and before blocking) for $90 \mathrm{~min}$ with the same concentrations of $\mathrm{KSCN}$ or urea used for the assays previously described. TfR binding was then performed under standard assay conditions.

\section{Verification of the Results from DOCILE by Elution from Transferrin-Sepharose}

A TfR-loaded transferrin-sepharose was prepared as previously described and aliquoted into $50-\mu \mathrm{L}$ portions. Each portion was washed twice with $500 \mu \mathrm{L}$ PBS and then mixed with $100 \mu \mathrm{L}$ of different elution buffers (see Figure 4). After 90 min incubation at $21^{\circ} \mathrm{C}$ with occasional mixing, the supernatant was dialyzed $3 \times 30$ min against PBS using micro-collodion bags (Sartorius AG, Göttingen, Germany). The samples were diluted with PBST to a final volume of $425 \mu \mathrm{L}$, and the TfR was quantified as follows. To determine the total amount of TfR, $100 \mu \mathrm{L}$ OKT9 (1 $\mathrm{mg} / \mathrm{mL}$ in PBS) were immobilized to Nunc-immuno modules MaxiSorp U16. After washing and blocking, $100 \mu \mathrm{L}$ of the samples (duplicates) were incubated at $21^{\circ} \mathrm{C}$ for $2.5 \mathrm{~h}$ and detected with polyclonal rabbit anti-TfR serum (dilut- 
ed 1:1000 with PBST) and enzyme-labeled porcine anti-rabbit IgG serum (diluted 1:2000 with PBST). Incubation periods and color development were as previously described. Purified and quantified TfR was used for calibration.

For quantitation of TfR capable of binding transferrin, an ELISA was performed in which $500 \mathrm{ng} /$ well ferritransferrin were immobilized and, after blocking, incubated with $100 \mu \mathrm{L}$ of the samples in duplicate. The detection of bound TfR was identical to that described for DOCILE.

\section{RESULTS}

\section{DOCILE}

To verify this new assay, we exam ined as a model the binding of the human TfR to its natural ligand transferrin. The TfR used for DOCILE revealed more than $95 \%$ rebinding activity when eluted with $\mathrm{KCl}$ from a ferri-transferrin affinity column (data not shown). Although $\mathrm{KCl}$ is known to be the best eluent for TfR from this column, we demonstrate here the applicability of the new assay for optimizing the elution with urea and KSCN because these compounds have the potential to irreversibly inactivate proteins and are, therefore, best suited to show the power of the new assay.

Although all experiments have been done by binding active $\mathrm{KCl}$-eluted TfR, it is not necessary for DOCILE to use a highly purified fraction with full binding activity. We showed that precise binding curves could also be generated using receptor fractions with a low content $(<5 \%)$ of binding active TfR. How ever, the amount of total TfR used per well had to be increased from 25 to 400 ng. Using a receptor preparation with high-binding activity, we were able to detect even $0.2 \mathrm{ng}$ TfR with high accuracy (absorbance was 5-fold above background). If the target protein is rare, the input amount of TfR can be reduced to $2 \mathrm{ng}$ without a serious loss in the quantitation of the binding activity.

\section{DOCILE with Urea}

To quantitate both the dissociation of a target protein (TfR) from an affini- ty ligand protein (transferrin) and the stability of the target protein in the presence of the eluent, two different experiments were performed for each assay, designated as elution and stability experiments, respectively. In the elution experiment, the binding of TfR to immobilized transferrin was measured in the presence of $0-3.5 \mathrm{M}$ urea (Figure $1 \mathrm{~A}$, open circles). Up to a concentration of $2.0 \mathrm{M}$ urea, the binding decreased only slightly and almost linearly from $100 \%$ to $88 \%$. Within the 2.0-2.62 $\mathrm{M}$ interval, the binding decreased rapidly to $5 \%$ in a sigmoidal pattern and, at higher concentrations of

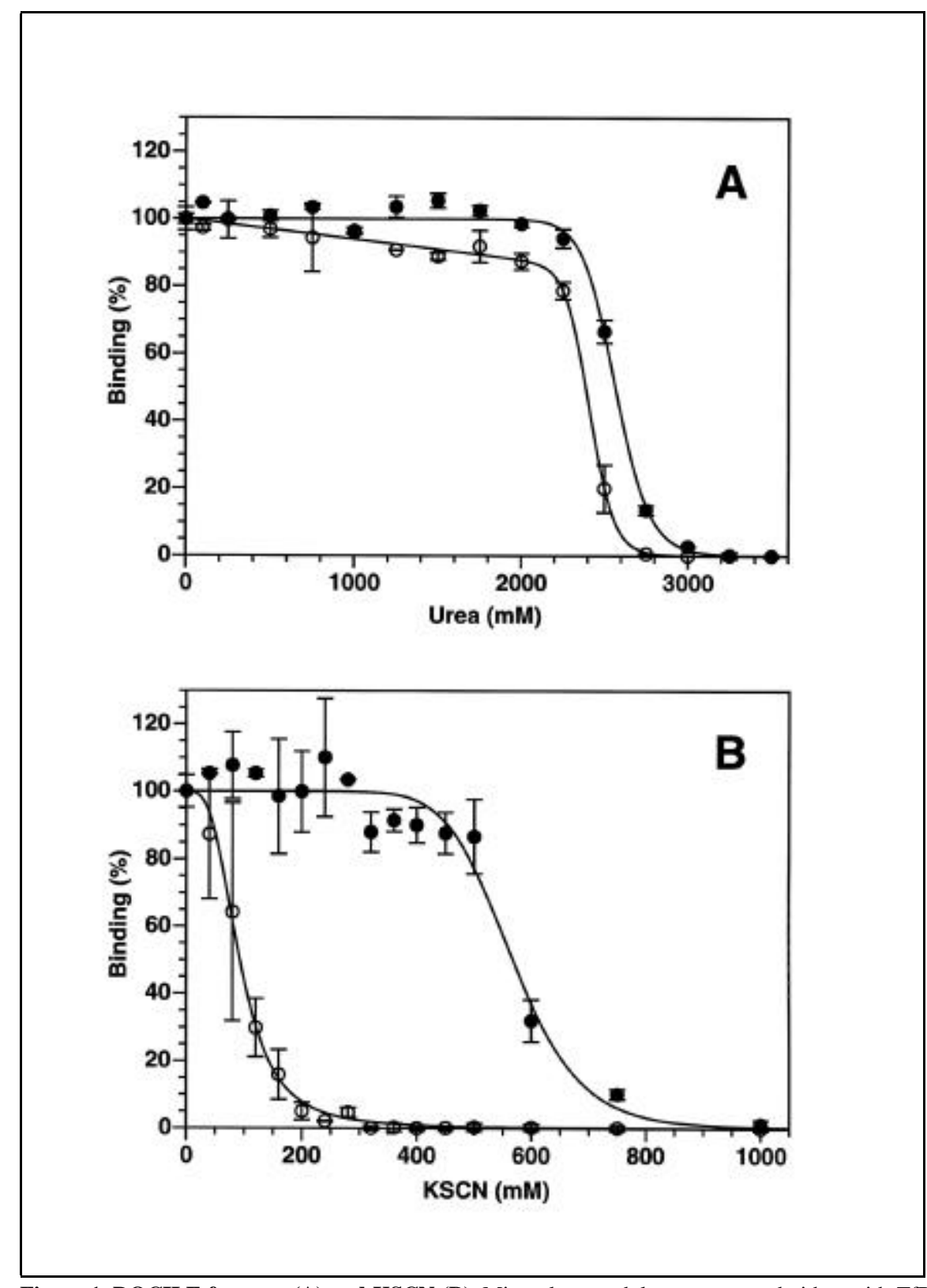

Figure 1. DOCILE for urea (A) and KSCN (B). Microplate modules were coated either with TfR (closed circles; stability experiment) or with transferrin (open circles; elution experiment). In the stability experiment, immobilized TfR was preincubated with increasing amounts of either urea (A) or KSCN (B). After the removal of the potential eluent, the binding of transferrin was quantitated in the ELISA described as part of the DOCILE. In the elution experiment, immobilized transferrin was incubated with TfR in the presence of the same increasing amounts of urea (A) or KSCN (B) used in the stability experiment. The amount of bound TfR was quantitated analogously to transferrin. To compare the results, the binding in absence of any eluent was set to $100 \%$. All data points represent the mean of two experiments. 
sigmoidal curve toward higher urea concentrations. Thus, it can be expected that an optimal elution concentration can be calculated. To ensure that the decreased signal at higher eluent concentrations is not caused by a release of coated proteins, the amount of coated protein after incubation with various eluent concentrations was quantitated by a direct detection with an antibody against the coated protein and found to be unchanged (data not shown). In the possible case that other coated proteins are actually released from the surface by the impact of the eluent, those proteins can be analogous to the covalent immobilization of proteins onto the matrix of an affinity column covalently immobilized to the microplate using an activated surface like CovaLink ${ }^{\mathrm{TM}}$ (Nalge Nunc International).

\section{DOCILE with KSCN and Other Eluents}

The second eluent that we investigated was KSCN. Similar to urea, both binding curves showed a sigmoidal decrease in binding for increasing concentrations of KSCN, but the shift toward higher concentrations in the stability curve was markedly increased in comparison to urea (Figure 1B). Thus, a concentration interval for complete elution with fully binding active receptor can be described.

We also examined $\mathrm{NaCl}$ and $\mathrm{KCl}$ as eluents (data not shown). These eluents did not result in any detectable binding activity loss in the stability experiment. The elution of $50 \%$ occurred at $1.32 \mathrm{M}$ $\mathrm{NaCl}$ or $585 \mathrm{mM} \mathrm{KCl}$, and elution of $95 \%$ occurred at $2.43 \mathrm{M} \mathrm{NaCl}$ or 2.02 $\mathrm{M} \mathrm{KCl}$. At $1 \mathrm{M} \mathrm{NaCl}$ or $\mathrm{KCl}, 28.0 \%$ or $78.2 \%$ TfR, respectively, could be eluted. Therefore, we purified the TfR by elution with $2 \mathrm{M} \mathrm{KCl}$. Interestingly, both eluents showed a slight, relative binding maximum at $150 \mathrm{mM}$ eluent, which is $8 \%$ higher binding than at 0 mM eluent (i.e., at conditions that mostly resemble the physiological state).

\section{Stability of the Affinity Matrix}

The immobilized protein can also be denatured by the eluent. This is important, if the affinity column is to be reused. Therefore, we also tested the sta- bility of transferrin in the presence of urea or KSCN. Figure 2A shows that urea did not affect the stability of transferrin up to $3.5 \mathrm{M}$. In contrast, $1 \mathrm{M}$ KSCN slightly decreased the stability of transferrin, resulting in only $93 \%$ TfR binding. Thus, a ferri-transferrin sepharose matrix is expected to lose binding capacity if reused after elution with KSCN.
The quantitation of the affinity matrix stability also allows one to determine whether the elution of a stable target protein was caused either by denaturation of the immobilized protein or suppression of the binding forces. In the latter case, the elution of an active target protein is possible only with concomitant loss of the binding capacity of the matrix.

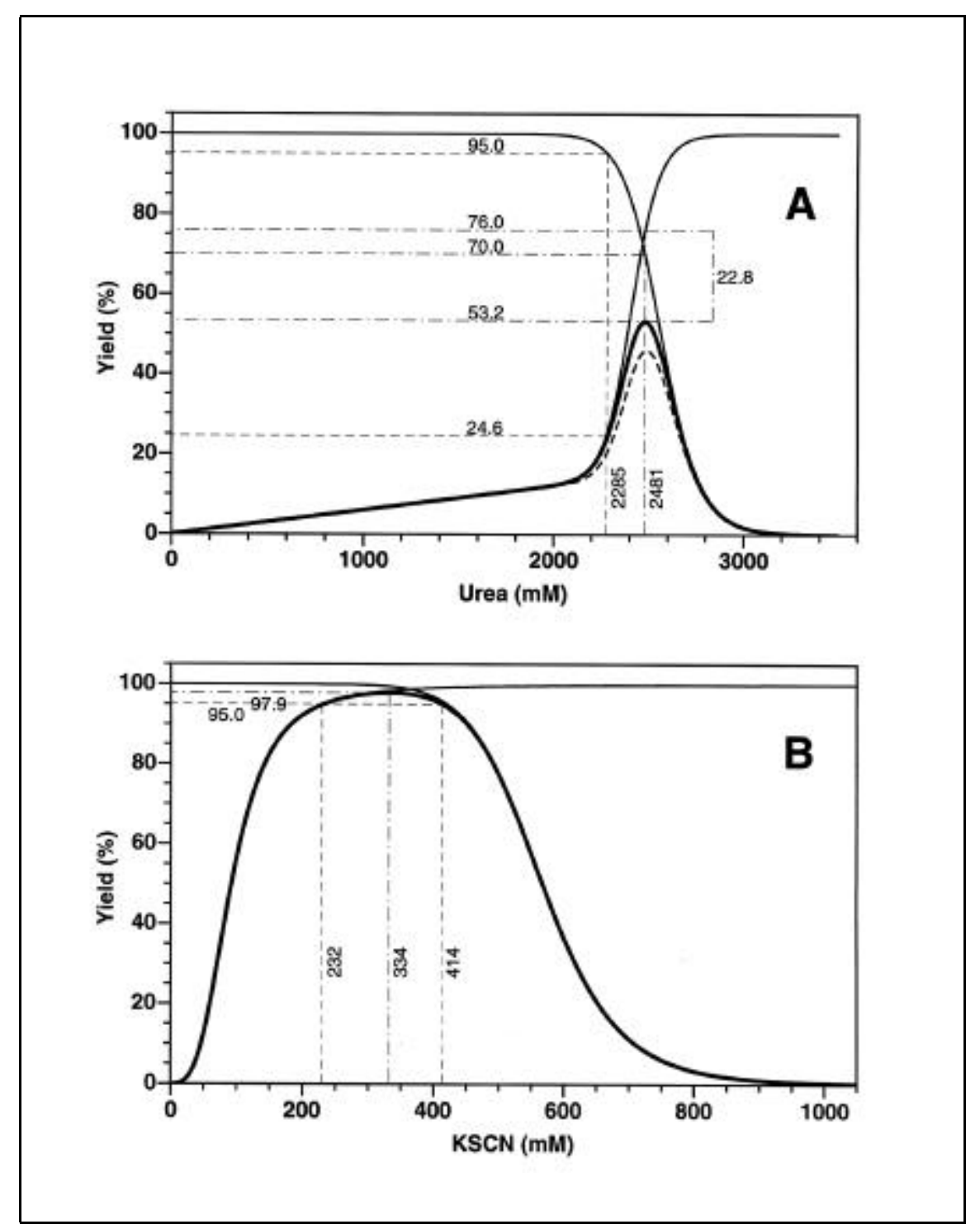

Figure 3. Calculation of optimal elution conditions for urea (A) and $\mathrm{KSCN}$ (B) from the data in Figure 1. The descending thin line shows the relative amount of binding active TfR (irrespective of whether it was eluted or not) from total TfR (irrespective of its binding activity). Assuming a stability-independent elution, this relative amount is identical to the amount of binding active TfR from eluted TfR. The ascending thin line shows the relative amount of eluted TfR (irrespective of its binding ability) from total TfR. Both thin curves were derived from the data in Figure 1. The thick line shows the relative amount of eluted binding active TfR from total TfR and was calculated as the product of the two thin curves. The dashed line represents the relative amount of eluted binding active TfR from total TfR, assuming that all inactive receptor molecules are part of the eluted fraction. Using $\operatorname{KSCN}(B)$, the difference between stability-dependent and independent elution was extremely subtle so that the dashed line was superimposed by the thick line. 


\section{Research Report}

\section{Calculation of Optimal Elution Conditions}

An optimal eluent concentration can be easily derived from the binding curves in Figure 1. If functionally active protein is not necessary (e.g., for sequence analyses), then any eluent concentration in which no binding was detected in the elution experiment can be chosen. Because it is unpredictable whether the percentage of active protein from total protein is identical with that from actually eluted protein, two general cases must be distinguished: (i) conformational changes of the protein do not influence its elution behavior (stability-independent elution) and (ii) the stability of the protein does affect its elution (stability-dependent elution). In the latter case, two boundary conditions are imaginable: (i) all inactive proteins will stick to the affinity matrix protein or to the affinity matrix itself, resulting in fully active eluted protein or (ii), that all inactive proteins completely elute from the matrix protein, resulting in a reduced fraction of active protein. Whereas the first boundary condition is not problematic because the eluted protein is fully active, the second more likely condition and the stability-independent elution are of great interest.

If elution and stability are independent events, then the fraction of eluted binding active protein from total pro- tein can be calculated as the product of the fraction of binding active protein (stability experiment) and eluted protein (elution experiment). Figure 3A shows the yield of binding active protein from urea-eluted TfR (thick line). At a urea concentration of $2.48 \mathrm{M}$, the yield of eluted binding active protein reached a maximum of $53.2 \%$ of total protein. However, only $70 \%$ of the total

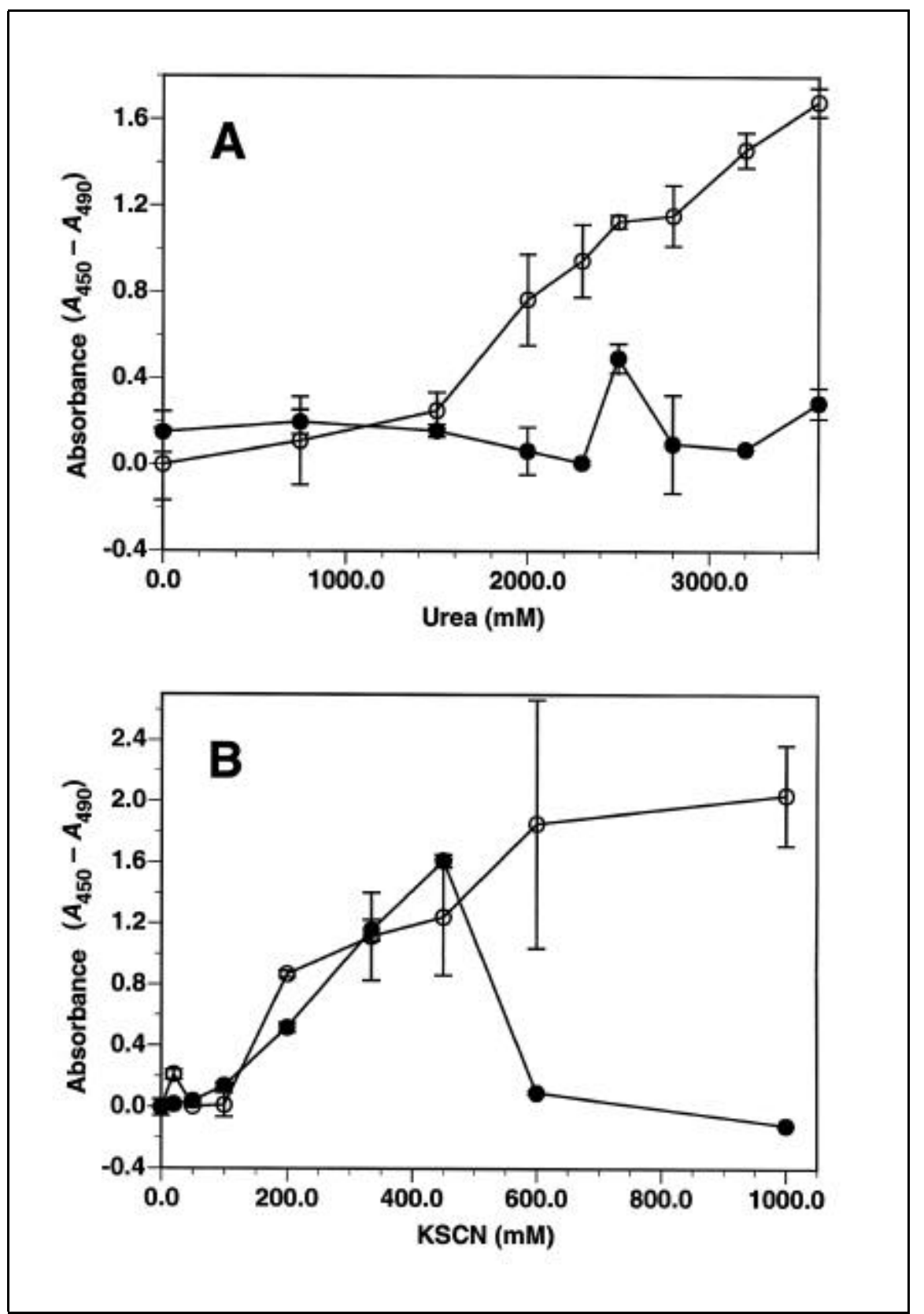

Figure 4. Elution of TfR from transferrin-sepharose by urea (A) and $\mathrm{KSCN}(\mathrm{B})$. TfR was eluted from transferrin-sepharose at various eluent concentrations. The amounts of total eluted TfR (irrespective of binding activity; open circles) and the amounts of eluted binding active TfR (closed circles) were determined for each sample after dialysis against PBS. All data points represent the mean of two experiments. 


\section{Research Report}

protein was active at this concentration (thin descending line). Thus, 30\% of the total protein (and therefore also $30 \%$ of the total eluted protein) was inactive $(53.2 \%$ was eluted and active; $22.8 \%$ was eluted, but inactive; $16.8 \%$ was not eluted, but active; and $7.2 \%$ was neither eluted nor active) (see Figure $3 \mathrm{~A}$ ). For many studies, a fraction of $30 \%$ inactive protein is not acceptable. DOCILE allows the determination of the optimal eluent concentration even if a relatively inappropriate eluent is available. Figure 3A shows that a urea concentration of less than $2.29 \mathrm{M}$ is required to achieve that the eluted protein contains at least $95 \%$ binding active protein (thin descending line). However, this high activity is contrasted with a mere $24.6 \%$ yield of eluted binding active protein from total protein, less than half of that obtained with $2.48 \mathrm{M}$ urea.

In an unfavorable case, all inactive proteins elute completely from the matrix. Figure 3A shows that in this case (dashed line) the maximum yield of eluted binding active protein was reduced to $46.0 \%$ and was reached at a concentration of $2.49 \mathrm{M}$ urea (thick line).

The same considerations for urea were also taken for KSCN (Figure 3B). Because the elution was nearly com plete at a KSCN concentration in which the binding activity was fully preserved, only a very subtle difference can be observed between the two elution models. Therefore, the concentrations of KSCN that yielded more than 95\% eluted binding active TfR (of total TfR) covered a wide range from 232 to $414 \mathrm{mM}$ (thick line) with a maximum at $334 \mathrm{mM}$ that yielded $97.9 \%$ eluted binding active receptor and only $0.5 \%$ eluted inactive TfR (the remainder was not eluted).

\section{Verification of DOCILE by TfR Elution from Transferrin-Sepharose}

To verify the predictions derived from DOCILE, the actual amount of total eluted and eluted binding active TfR from transferrin-sepharose was determined. Figure 4A shows that the amount of total eluted TfR (open circles) increased with increasing amounts of urea, whereas the amount of eluted binding active TfR (closed circles) reached a clearly visible but not prominent maximum between 2.3 and $2.8 \mathrm{M}$. These results are in direct agreement with the data predicted by DOCILE (compare with Figure 3A; thick line and ascending thin line). The actual results obtained by KSCN elution from transferrin-sepharose (Figure 4B) also correspond almost perfectly with the predictions derived from DOCILE (compare with Figure 3B; thick line and ascending thin line). The broad and prominent peak (closed circles) shows that complete elution of binding active receptor with $\mathrm{KSCN}$ is possible, provided that the eluent concentration is carefully chosen.

\section{DISCUSSION}

In the present study, we have developed a new assay for the determination of optimal conditions for ligand elution. The importance of this assay may increase because today's proteins of interest are often very rare molecules. Because a gentle elution often leads to an incomplete release of the target protein and strong elution conditions may functionally inactivate the target protein or the affinity matrix, DOCILE is an interesting tool that can be used to find optimal elution conditions with only a tiny consumption of the target protein.

An apparent disadvantage of DOCILE is the requirement of antibodies against the affinity matrix and target proteins. However, commercial antibodies are available against most of the high-affinity matrix proteins. If antibodies are unavailable, both the target and the matrix protein can be labeled (e.g., by iodination, biotinylation, etc.) for subsequent quantitation.

Before performing DOCILE, the time course and temperature of the planned preparative purification should be known. The temperature should be kept constant throughout. In the stability experiment, it is furthermore recommended to include a preincubation period of length similar to the expected eluent exposure time required for the planned affinity chromatography.

Another important aspect is whether the planned elution of the protein to be purified will occur by batch or from a column because DOCILE elution ex- 
periment resembles elution by batch. Whereas for a batch experiment both the association and the dissociation kinetics are important, for the elution from a column only the dissociation kinetics has a decisive influence. For an accurate prediction, the rate constants can easily be calculated using a direct calibration ELISA (5). As soon as the equilibrium is reached in a batch elution, the conditions are identical to those determined in the elution experiment of DOCILE. In contrast, elution from a column is a non-equilibrium situation. The decrease in affinity at higher eluent concentrations can be caused by an increased dissociation rate or a decreased association rate. In the latter case, the elution might require either higher eluent concentrations than predicted by DOCILE or a very slow elution flow and a flat gradient. Of course, it is possible to determine a suitable elution concentration by performing several gradient elutions from a column, but more material is required, much of the target protein may be inactive, and the possibility of testing a large set of different eluents is extremely limited.

The precision of DOCILE can be further demonstrated by the comparison of the predicted data for $\mathrm{NaCl}$ and $\mathrm{KCl}$ elutions with experiments described in the literature. Seligman et al. (10) first described the elution of TfR with $1 \mathrm{M} \mathrm{NaCl}$. However, Turkewitz et al. (11) found that under these conditions, only a small fraction of the bound receptor elutes $(28 \%$ predicted by DOCILE). Anderson et al. (1) reported the use of $1 \mathrm{M} \mathrm{KCl}$ for the elution of TfR from transferrin-sepharose $(78 \%$ predicted by DOCILE). Turkewitz et al. (11) reproduced this experiment but found that rapid and efficient elution is more easily achieved using $2 \mathrm{M} \mathrm{KCl}$ (95\% predicted by DOCILE).

Proteins can possess functions other than ligand binding (e.g., enzymatic activity). It cannot be predicted from DOCILE whether the eluted binding active protein will also preserve these additional functions. However, slight modifications of the assay may be sufficient to answer these questions. For instance, in an additional experiment, the enzymatic activity of a surfacebound protein can be screened with a chromogenic substrate subsequent to the preincubation with the eluent.

In summary, DOCILE is a promising tool for the determination of optimal elution conditions for affinity chromatography. Furthermore, the stability of the matrix protein can also be quantitated to avoid the loss of column capacity. Because only tiny amounts of the target protein are necessary for a complete elution analysis, DOCILE may become an important assay for the purification of rare proteins.

\section{ACKNOWLEDGMENTS}

We thank the Deutsche Forschungsgemeinschaft for financial support (Sonderforschungsbereich 312, Teilprojekt D4).

\section{REFERENCES}

1.Anderson, G.J., A. Mackerras, L.W. Powell, and J.W. Halliday. 1986. Improved purification of the human placental transferrin receptor and a novel immunoradiometric assay for receptor protein. Biochim. Biophys. Acta 884:225-233.

2.Chitambar, C.R. and Z. Zivkovic. 1989. Release of soluble transferrin receptor from the surface of human leukemic HL60 cells. Blood 74:602-608.

3.Dautry-Varsat, A., A. Ciechanover, and H.F. Lodish. 1983. pH and the recycling of transferrin during receptor-mediated endocytosis. Proc. Natl. Acad. Sci. USA 80:22582262.

4.Fuchs, H., R. Geßner, R. Tauber, and R. Ghosh. 1995. Functional reconstitution of the human placental transferrin receptor into phospholipid bilayers leads to long tubular structures proceeding from the vesicle surface. Biochemistry 34:6196-6207.

5.Fuchs, H., G. Orberger, R. Tauber, and R. Gessner. 1995. Direct calibration ELISA: a rapid method for the simplified determination of association constants of unlabeled biological molecules. J. Immunol. Methods 188:197208.

6.Gallati, H. and I. Pracht. 1985. Horseradish peroxidase: kinetic studies and optimization of peroxidase activity determination using the substrates $\mathrm{H} 2 \mathrm{O} 2$ and 3,3',5,5'-tetramethylbenzidine. J. Clin. Chem. Clin. Biochem. 23:453460.

7.Hopkins, C.R., A. Gibson, M. Shipman, D.K. Strickland, and I.S. Trowbridge. 1994. In migrating fibroblasts, recycling receptors are concentrated in narrow tubules in the pericentriolar area, and then routed to the plasma membrane of the leading lamella. J. Cell Biol. 125:1265-1274.

8.Jing, S.Q. and I.S. Trowbridge. 1987. Identi- fication of the intermolecular disulfide bonds of the human transferrin receptor and its lipidattachment site. EMBO J. 6:327-331.

9.Karin, M. and B. Mintz. 1981. Receptor-mediated endocytosis of transferrin in developmentally totipotent mouse teratocarcinoma stem cells. J. Biol. Chem. 256:3245-3252.

10.Seligman, P.A., R.B. Schleicher, and R.H. Allen. 1979. Isolation and characterization of the transferrin receptor from human placenta. J. Biol. Chem. 254:9943-9946.

11.Turkewitz, A.P., J.F. Amatruda, D. Borhani, S.C. Harrison, and A.L. Schwartz. 1988. A high yield purification of the human transferrin receptor and properties of its major extracellular fragment. J. Biol. Chem. 263:83188325 .

Received 29 November 2000; accepted 4 April 2001.

\author{
Address correspondence to: \\ Dr. Hendrik Fuchs \\ Institut für Klinische Chemie und Pathobiochemie \\ Universitätsklinikum Benjamin Franklin \\ Freie Universität Berlin \\ Hindenburgdamm 30 \\ D-12200 Berlin, Germany \\ e-mail: hendrik.fuchs@ukbf.fu-berlin.de
}

For reprints of this or
any other article,contact
Reprints@BioTechniques.com

